\title{
Armazenamento de sementes de feijão revestidas com polímeros e tratadas com fungicidas
}

\author{
Larissa Leandro Pires ${ }^{(1)}$, Cláudio Bragantini(2) e Jefferson Luís da Silva Costa ${ }^{(3)}$ \\ (1)Universidade Federal de Goiás, Campus Samambaia, Caixa Postal 131, CEP 74001-970 Goiânia, GO. E-mail: larissaleandropires@bol.com.br \\ (2)Embrapa Arroz e Feijão, Caixa Postal 179, CEP 75375-000 Santo Antônio de Goiás, GO. E-mail: claudio@cnpaf.embrapa.br (3)Embrapa \\ Tabuleiros Costeiros, Av. Beira Mar, 3250, CEP 49025-040 Aracaju, SE. E-mail: jcosta@cpatc.embrapa.br
}

\begin{abstract}
Resumo - O revestimento com polímeros, associado ao tratamento químico de sementes, vem sendo utilizado em culturas com o propósito de fixar produtos químicos. O objetivo do trabalho foi avaliar o efeito do armazenamento na qualidade de sementes de feijão (Phaseolus vulgaris L.) revestidas com polímeros e tratadas com diferentes fungicidas. As sementes foram inoculadas por imersão a vácuo, em suspensão de inóculo de Colletotrichum lindemuthianum $\left(5 \times 10^{5}\right.$ conídios $\left./ \mathrm{mL}\right)$, fungo-teste utilizado. Os fungicidas benomyl, carbendazin e captan, nas formulações pó molhável, suspensão concentrada e pó seco, respectivamente, foram aplicados prévia ou concomitantemente à aplicação de uma mistura de dois polímeros à base de tintas de polímeros vinílicos fosca e brilhante. Apesar de a velocidade de germinação das sementes de feijão revestidas e tratadas ter sido reduzida, a porcentagem de germinação não foi afetada negativamente pelo revestimento com os polímeros testados. $\mathrm{O}$ revestimento com polímeros não prejudicou a eficiência dos fungicidas testados no controle de fungos em geral, sob nenhuma das duas formas de aplicação, ao longo dos quatro meses de armazenamento. O revestimento com estes polímeros pode afetar a eficiência de controle de patógenos nestas sementes, positiva ou negativamente, dependendo do fungicida utilizado e de sua forma de aplicação e dependendo do patógeno presente nas sementes.
\end{abstract}

Termos para indexação: Phaseolus vulgaris, revestimento, tratamento químico.

\section{Storage of dry bean seeds coated with polymers and treated with fungicides}

\begin{abstract}
Coating seeds with polymers associated with chemicals is used to promote a better fixation of seed treatment products, and therefore minimizing toxic hazards. The objective of this work was to verify the effect of four-month storage on quality of dry bean (Phaseolus vulgaris L.) seed coated with polymers, and treated with fungicides. Bean seeds were inoculated by immersion in a suspension of Colletotrichum lindemuthianum $\left(5 \times 10^{5}\right.$ conidia $\left./ \mathrm{mL}\right)$, applied under vacuum. After the inoculation, seeds were treated with different types of fungicides: wettable powder, dry powder and concentrated suspension, applied either mixed or prior to polymer coating. Two different types of PVA paint were used as coating agent. Seed quality was evaluated through germination, vigor and pathological tests. Germination of coated and treated seeds was not affected during the storage period. The association of coating and a wettable powder treatment showed a higher germination percentage after two months of storage. The formulations wettable powder and concentrated suspension promoted a better control of the pathogens.
\end{abstract}

Index terms: Phaseolus vulgaris, coating, seed treatment.

\section{Introdução}

O feijoeiro é uma cultura suscetível a inúmeras doenças causadas por fungos, bactérias e vírus, transmitidos interna e externamente às sementes, e, por isso, a obtenção de sementes com alta qualidade sanitária e fisiológica tem sido limitada. A gravidade dos danos causados por esses patógenos torna o interesse pelo tratamento químico de sementes crescente, de modo a diminuir a quantidade de inóculo associado às sementes
(Azevedo, 1996; Sartorato, 1996). Entretanto, o uso de fungicidas em sementes de feijoeiro constitui, ainda, uma técnica pouco investigada sob o aspecto de sua eficiência, distribuição, uniformidade de cobertura e capacidade de aderência (Azevedo, 1996).

Nos últimos anos, o tratamento químico de sementes que utiliza o revestimento com polímeros tem recebido atenção em algumas culturas de expressão econômica. Os polímeros têm possibilitado o aumento da penetração e da fixação do produto ativo, melhorando, conse- 
qüentemente, a sua distribuição nas sementes, além de reduzir as quantidades utilizadas de produtos químicos e os problemas de poluição ambiental (Duran, 1998). O revestimento ainda proporciona uma cobertura durável, permeável à água, com a possibilidade de aplicação em sementes de diferentes formas e tamanhos, sem afetar seu processo germinativo (Bacon \& Clayton, 1986; Maude, 1998).

Sementes contendo inseticidas e fungicidas, em formulações líquida, pó ou suspensão concentrada, dissolvidos ou dispersos na formulação do produto de revestimento, já podem ser comercializadas. Entretanto, existe pouca informação sobre o revestimento, desde os próprios polímeros a serem utilizados, com suas respectivas dosagens, até a tecnologia adequada ao tratamento de grandes volumes (Maude, 1998).

O objetivo deste trabalho foi avaliar o efeito do armazenamento na qualidade de sementes de feijão revestidas com polímeros e tratadas com diferentes fungicidas.

\section{Material e Métodos}

Sementes de feijão (Phaseolus vulgaris L.) da cultivar Pérola, suscetível à antracnose (Lanza et al., 1997), foram submetidas à inoculação artificial de Colletotrichum lindemuthianum (isolado Cl CNF 540, patótipo 89). Este fungo foi utilizado como fungo-teste, por ser de ocorrência comum e possuir um método de inoculação bem definido e com resultados consistentes.

O fungo foi cultivado em meio batata-dextrose-ágar (BDA), e mantido à temperatura de $21,1^{\circ} \mathrm{C}$ por sete dias. Em seguida, foi repicado para vagens de feijão previamente esterilizadas, parcialmente embebidas em BDA, e mantidas em incubadora à temperatura de $21,1^{\circ} \mathrm{C}$ por sete dias. Após o período de incubação, as vagens foram maceradas em água (Cárdenas et al., 1964), ajustando-se a suspensão de esporos obtida para $5 \times 10^{5}$ conídios $/ \mathrm{mL}$ de água destilada e esterilizada.

A inoculação foi realizada pelo método de infiltração a vácuo, imergindo $1,5 \mathrm{~kg}$ de sementes em $2,0 \mathrm{~L}$ da suspensão de esporos, dentro da cúpula de uma bomba de vácuo, por um período de 1,5 minuto. Em seguida, as sementes foram postas a secar à sombra, ao ar livre, por 24 horas.

Após a secagem, as sementes foram revestidas com uma mistura de duas tintas de polímeros vinílicos: uma fosca e outra brilhante $(4,7 \mathrm{~mL}+4,7 \mathrm{~mL}$ de produto comercial $/ \mathrm{kg}$ de sementes), na concentração de $100 \%$.

Três fungicidas de diferentes formulações foram testados: carbendazin (suspensão concentrada, 1,0 mL/kg de sementes), benomyl (pó molhável, $1,0 \mathrm{~g} / \mathrm{kg}$ de sementes) e captan (pó seco, 1,0 g/kg de sementes). Os fungicidas foram aplicados às sementes sob duas formas: concomitantemente ou antes da aplicação dos polímeros. Como controles, foram utilizadas sementes com e sem inoculação, ambas sem aplicação de fungicidas e de polímeros. Em todos os tratamentos, foram adicionados 2,0 $\mathrm{mL}$ de corante alimentício de cor roxa por $\mathrm{kg}$ de sementes, como indicador do recobrimento.

Para a realização de cada tratamento, utilizou-se uma amostra de $500 \mathrm{~g}$ de sementes de feijão, colocada em uma máquina tratadora de sementes, do tipo betoneira, em movimento rotativo por cinco minutos. Em seguida, as sementes foram postas a secar à sombra, ao ar livre, durante 24 horas e, posteriormente, armazenadas em caixas de papelão em condições ambientais de laboratório, por um período de quatro meses.

A avaliação do efeito do armazenamento na qualidade de sementes de feijão foi efetuada logo após o revestimento ou tratamento químico e ao longo do armazenamento (um, dois e quatro meses), mediante teste de germinação e teste de sanidade de sementes, conforme as regras para análises de sementes (Brasil, 1992). As sementes foram submetidas às análises de germinação pelo método do rolo de papel toalha, obtendo-se a porcentagem de plântulas normais aos sete dias (germinação), e o vigor foi avaliado indiretamente pela velocidade de germinação, utilizando-se como parâmetro a primeira contagem de plântulas normais do teste de germinação, realizada aos quatro dias.

$\mathrm{O}$ teste de sanidade de sementes foi conduzido pelos métodos do rolo de papel e de papel de filtro com congelamento, nos quais procedeu-se à identificação dos patógenos e contagem de sementes infectadas e de plântulas com sintomas e sinais dos patógenos nos cotilédones e nas radículas. No método do rolo de papel, 50 sementes, envolvidas por folhas de papel toalha umedecidas com água destilada e esterilizada, acondicionadas em sacos de plástico escuro perfurados, foram incubadas à temperatura de $20 \pm 2^{\circ} \mathrm{C}$ por sete dias. Pelo método de papel de filtro com congelamento, 20 sementes, acondicionadas em caixa gerbox contendo folhas de papel de filtro umedecidas com água destilada e esterilizada, foram colocadas em câmara de incubação à temperatura de $20 \pm 2^{\circ} \mathrm{C}$ por 24 horas, em regime de luz e escuro alternado, de 12 horas. Em seguida, as sementes foram submetidas ao congelamento $\left(-20^{\circ} \mathrm{C}\right)$ por 24 horas. Após o término deste período, as sementes retornaram à câmara de incubação, onde permaneceram por mais sete dias, visando ao adequado desenvolvimento dos patógenos. 
O delineamento adotado foi o de parcelas subdivididas no tempo, e os tratamentos constituíram-se de parcelas e subparcelas representadas pelas épocas de armazenamento (zero, um, dois e quatro meses) avaliadas. Foram realizadas as análises de variância e comparação de médias, pelo teste de Scott-Knott (Scott \& Knott, 1974). As variáveis porcentagem de Colletotrichum lindemuthianum, de Eurotium sp., de Cladosporium sp., de Penicillium sp., de Aspergillus sp., de Rhizopus sp., de Fusarium sp., de Alternaria sp. e de bactérias foram transformadas em arco seno $\sqrt{\%}$. O experimento foi repetido duas vezes.

\section{Resultados e Discussão}

$\mathrm{O}$ vigor, avaliado pela primeira contagem de germinação, das sementes de feijão tratadas com fungicidas e revestidas com polímeros não superou o das sementes tratadas apenas com fungicidas, principalmente até o primeiro mês de armazenamento (Tabela 1). Contrariamente, Bacon \& Clayton (1986) verificaram emergência simultânea das sementes de cevada revestidas com produto solúvel em água e tratadas com fungicida em pó, e do controle. Sementes tratadas apenas com o fungicida mostraram um atraso relativo de dois a três dias na emergência.

Apesar deste retardamento no início do processo germinativo, a germinação e o desenvolvimento normal das plântulas não foram afetados negativamente pelo revestimento durante os quatro meses de armazenamento, independentemente da forma de aplicação e do fungicida testado (Tabela 1). As sementes tratadas com benomyl (pó molhável) e com carbendazin (suspensão concentrada) e revestidas com polímeros mostraram, até mesmo, maior porcentagem de germinação do que as que receberam somente o tratamento com esses fungicidas, após dois meses de armazenamento. Como o teste de vigor utilizado avalia a velocidade de germinação das sementes e essa é dependente da rapidez com que elas absorvem água para iniciar o processo (Bewley \& Black, 1985), acredita-se que as diferenças em vigor encontradas não caracterizem diferenças em qualidade fisiológica das sementes revestidas e, sim, diferenças quanto à sua capacidade de absorver água, efeito acentuado, especialmente, imediatamente após a aplicação dos polímeros.

As sementes tratadas com o fungicida captan (pó seco), associado ou não aos polímeros, mostraram menor vigor, principalmente após dois meses de armazenamento, em relação às sementes tratadas com os fungicidas benomyl e carbendazin. Contudo, esta diferença não foi verificada em termos de germinação (Tabela 1).

Os fungos detectados nas sementes de feijão durante os quatro meses de armazenamento foram: Colletotrichum lindemuthianum e Eurotium sp., pelo método do rolo de papel; pelo método do papel de filtro com congelamento foram melhor detectados Aspergillus sp., Penicillium sp., Fusarium sp.,

Tabela 1. Vigor e germinação de sementes de feijão (Phaseolus vulgaris L.), revestidas com polímeros e tratadas com diferentes fungicidas, antes e durante o amarzenamento ${ }^{(1)}$.

\begin{tabular}{|c|c|c|c|c|c|c|c|c|}
\hline \multirow[t]{3}{*}{ Tratamento $^{(2)}$} & \multicolumn{4}{|c|}{ Vigor (\%) } & \multicolumn{4}{|c|}{ Germinação (\%) } \\
\hline & \multicolumn{4}{|c|}{ Período de armazenamento (mês) } & \multicolumn{4}{|c|}{ Período de armazenamento (mês) } \\
\hline & Antes & 1 & 2 & 4 & Antes & 1 & 2 & 4 \\
\hline $\mathrm{T}$ & $50,75 \mathrm{Bd}$ & $59,25 \mathrm{Cc}$ & $74,25 \mathrm{Aa}$ & $64,00 \mathrm{Ab}$ & $85,75 \mathrm{Ba}$ & $79,25 \mathrm{Ba}$ & $81,50 \mathrm{Aa}$ & $81,00 \mathrm{Aa}$ \\
\hline $\mathrm{TN}$ & $54,75 \mathrm{Bc}$ & $77,75 \mathrm{Aa}$ & $71,50 \mathrm{Aa}$ & $64,50 \mathrm{Ab}$ & $95,50 \mathrm{Aa}$ & $91,75 \mathrm{Aa}$ & $83,25 \mathrm{Ab}$ & $82,25 \mathrm{Ab}$ \\
\hline $\mathrm{P}$ & $42,25 \mathrm{Cb}$ & $57,75 \mathrm{Ca}$ & $62,25 \mathrm{Ba}$ & $61,50 \mathrm{Aa}$ & $85,50 \mathrm{Ba}$ & $82,00 \mathrm{Ba}$ & $83,25 \mathrm{Aa}$ & $74,00 \mathrm{Bb}$ \\
\hline B & $64,75 \mathrm{Ab}$ & $77,75 \mathrm{Aa}$ & $66,50 \mathrm{Ab}$ & $59,75 \mathrm{Ab}$ & $89,75 \mathrm{Aa}$ & $87,75 \mathrm{Aa}$ & $75,25 \mathrm{Bb}$ & $68,00 \mathrm{Cc}$ \\
\hline $\mathrm{B}+\mathrm{P}$ & $46,50 \mathrm{Cc}$ & $58,50 \mathrm{Cb}$ & $71,75 \mathrm{Aa}$ & $61,00 \mathrm{Ab}$ & $92,75 \mathrm{Aa}$ & $88,25 \mathrm{Ab}$ & $85,75 \mathrm{Ab}$ & $77,25 \mathrm{Ac}$ \\
\hline BMP & $38,75 \mathrm{Cc}$ & $68,25 \mathrm{Ba}$ & $67,25 \mathrm{Aa}$ & $60,25 \mathrm{Ab}$ & $89,75 \mathrm{Aa}$ & $88,00 \mathrm{Aa}$ & $84,00 \mathrm{Aa}$ & $78,00 \mathrm{Ab}$ \\
\hline $\mathrm{C}$ & $44,25 \mathrm{Cc}$ & $68,00 \mathrm{Ba}$ & $64,50 \mathrm{Ba}$ & $54,25 \mathrm{Bb}$ & $90,25 \mathrm{Aa}$ & $90,25 \mathrm{Aa}$ & $85,50 \mathrm{Aa}$ & $78,50 \mathrm{Ab}$ \\
\hline $\mathrm{C}+\mathrm{P}$ & $46,25 \mathrm{Cb}$ & $57,50 \mathrm{Ca}$ & $56,00 \mathrm{Ba}$ & $50,50 \mathrm{Bb}$ & $87,75 \mathrm{Ba}$ & $87,50 \mathrm{Aa}$ & $86,75 \mathrm{Aa}$ & $78,50 \mathrm{Ab}$ \\
\hline CMP & $25,50 \mathrm{Dc}$ & $53,75 \mathrm{Cb}$ & $60,25 \mathrm{Ba}$ & $49,75 \mathrm{Bb}$ & $85,25 \mathrm{Ba}$ & $87,50 \mathrm{Aa}$ & $85,25 \mathrm{Aa}$ & $80,25 \mathrm{Aa}$ \\
\hline $\mathrm{D}$ & $57,75 \mathrm{Bc}$ & $75,75 \mathrm{Aa}$ & $68,25 \mathrm{Ab}$ & $67,50 \mathrm{Ab}$ & $92,00 \mathrm{Aa}$ & $85,25 \mathrm{Ab}$ & $77,50 \mathrm{Bc}$ & $76,00 \mathrm{Ac}$ \\
\hline $\mathrm{D}+\mathrm{P}$ & $44,50 \mathrm{Cc}$ & $66,25 \mathrm{Ba}$ & $68,00 \mathrm{Aa}$ & $54,50 \mathrm{Bb}$ & $90,00 \mathrm{Aa}$ & $86,00 \mathrm{Aa}$ & $83,50 \mathrm{Aa}$ & $74,00 \mathrm{Bb}$ \\
\hline DMP & $43,25 \mathrm{Cc}$ & $68,50 \mathrm{Ba}$ & $67,25 \mathrm{Aa}$ & $56,75 \mathrm{Bb}$ & $90,25 \mathrm{Aa}$ & $85,75 \mathrm{Aa}$ & $81,00 \mathrm{Ab}$ & $76,50 \mathrm{Ab}$ \\
\hline
\end{tabular}

${ }^{(1)}$ Médias seguidas pela mesma letra, maiúscula na coluna (entre tratamentos) e minúscula na linha (entre épocas), não diferem entre si pelo teste de Scott-Knott, a 5\% de probabilidade; o CVa dos dados de vigor foi 7,96\% e o CVb, 7,45\%; o CVa dos dados de germinação foi 4,56\% e o CVb, 4,49\%. (2)T: controle com inoculação; TN: controle sem inoculação; P: polímero; B: benomyl (pó molhável); B+P: benomyl aplicado antes dos polímeros; BMP: benomyl misturado aos polímeros; C: captan (pó seco); C+P: captan aplicado antes dos polímeros; CMP: captan misturado aos polímeros; D: carbendazin (suspensão concentrada); D+P: carbendazin aplicado antes dos polímeros; DMP: carbendazin misturado aos polímeros. 
Alternaria sp., Rhizopus sp., Cladosporium sp. e Peztalotia sp., de ocorrência natural no lote de sementes utilizado.

O revestimento com polímeros não prejudicou a eficiência dos fungicidas testados no controle de fungos, sob nenhuma das duas formas de aplicação, ao longo do armazenamento (Tabela 2). Resultados semelhantes foram constatados em sementes de outras culturas (Sauve \& Shiel, 1980; Bacon \& Clayton, 1986). Aos quatro meses, a associação de benomyl e de carbendazin com o revestimento proporcionou aumento de sua eficiência no controle de patógenos, em relação às sementes tratadas somente com esses fungicidas. Bacon \& Clayton (1986) obtiveram resultados similares em sementes de cevada.

Os polímeros não afetaram a eficiência dos fungicidas testados no controle de $C$. lindemuthianum. Carbendazin e benomyl, associados ou não aos polímeros, controlaram este patógeno de maneira mais eficiente do que captan, no decorrer do armazenamento (Tabela 3).

A porcentagem de sementes com bactérias (método do rolo de papel) aumentou ao longo do armazenamento das sementes, o que pode ter mascarado os efeitos dos tratamentos químicos, e pode ter sido uma das causas

Tabela 2. Porcentagem de sementes de feijão (Phaseolus vulgaris $\mathrm{L}$.) livres de patógenos, após o revestimento com polímeros e tratamento com fungicidas, antes e durante o $\operatorname{armazenamento}^{(1)}$.

\begin{tabular}{lcccr}
\hline Tratamento $^{(2)}$ & \multicolumn{4}{c}{ Período de armazenamento (mês) } \\
\cline { 2 - 5 } & Antes & 1 & 2 & 4 \\
\hline B & $38,75 \mathrm{Ba}$ & $35,50 \mathrm{Aa}$ & $30,50 \mathrm{Aa}$ & $14,00 \mathrm{Cb}$ \\
B+P & $41,00 \mathrm{Ba}$ & $35,75 \mathrm{Aa}$ & $28,50 \mathrm{Ab}$ & $23,50 \mathrm{Bb}$ \\
BMP & $44,75 \mathrm{Ba}$ & $38,75 \mathrm{Ab}$ & $36,00 \mathrm{Ab}$ & $31,75 \mathrm{Ab}$ \\
C & $28,50 \mathrm{Ca}$ & $25,75 \mathrm{Ba}$ & $28,25 \mathrm{Ba}$ & $25,25 \mathrm{Ba}$ \\
C+P & $30,00 \mathrm{Ca}$ & $29,50 \mathrm{Ba}$ & $17,25 \mathrm{Bb}$ & $22,50 \mathrm{Bb}$ \\
CMP & $28,50 \mathrm{Ca}$ & $31,25 \mathrm{Ba}$ & $30,00 \mathrm{Aa}$ & $24,25 \mathrm{Ba}$ \\
D & $43,50 \mathrm{Ba}$ & $35,25 \mathrm{Ab}$ & $31,25 \mathrm{Ab}$ & $17,25 \mathrm{Cc}$ \\
D+P & $40,75 \mathrm{Ba}$ & $40,25 \mathrm{Aa}$ & $35,00 \mathrm{Aa}$ & $28,25 \mathrm{Ab}$ \\
DMP & $70,50 \mathrm{Aa}$ & $36,00 \mathrm{Ab}$ & $28,50 \mathrm{Ab}$ & $33,75 \mathrm{Ab}$ \\
P & $19,25 \mathrm{Da}$ & $12,75 \mathrm{Ca}$ & $23,25 \mathrm{Ba}$ & $18,00 \mathrm{Ca}$ \\
T & $17,00 \mathrm{Da}$ & $12,25 \mathrm{Cb}$ & $19,50 \mathrm{Ba}$ & $9,00 \mathrm{Cb}$ \\
TN & $40,50 \mathrm{Ba}$ & $42,50 \mathrm{Aa}$ & $32,75 \mathrm{Ab}$ & $31,75 \mathrm{Ab}$ \\
\hline
\end{tabular}

${ }^{(1)}$ Médias seguidas pela mesma letra, maiúscula na coluna (entre tratamentos) e minúscula na linha (entre épocas), não diferem entre si pelo teste de Scott-Knott, a 5\% de probabilidade; o CVa dos dados foi $18,58 \%$ e o $\mathrm{CVb}, 18,16 \%$. ${ }^{(2)} \mathrm{T}$ : controle com inoculação; TN: controle sem inoculação; P: polímero; B: benomyl (pó molhável); B+P: benomyl aplicado antes dos polímeros; BMP: benomyl misturado aos polímeros; C: captan (pó seco); C+P: captan aplicado antes dos polímeros; CMP: captan misturado aos polímeros; D: carbendazin (suspensão concentrada); D+P: carbendazin aplicado antes dos polímeros; DMP: carbendazin misturado aos polímeros. de redução da germinação das sementes (Tabelas 1 e 4), conforme também verificado por Menezes \& Mohan (1982). Para Christensen (1957), no entanto, as bactérias não parecem estar envolvidas na deterioração de sementes armazenadas, por exigirem água livre para o seu crescimento, condição raramente existente no ambiente de armazenamento.

O fato de as sementes terem sido revestidas não contribuiu para o desenvolvimento de bactérias (Tabela 4). Ao contrário, as sementes que foram revestidas com polímeros, independentemente da forma de aplicação junto aos fungicidas benomyl e carbendazin, apresentaram menor incidência de bactérias, ao longo do armazenamento, comparadas aos demais tratamentos e ao controle com inoculação. Contudo, nas sementes revestidas e tratadas com captan, a incidência de bactérias foi maior do que nas sementes apenas tratadas com esse fungicida.

Quanto à presença do fungo de armazenamento Aspergillus sp., as sementes tratadas e revestidas apresentaram porcentagens de infestação menores que $0,40 \%$, estatisticamente semelhantes entre si, e inferiores aos controles com e sem inoculação, até dois meses de armazenamento. Após este período, este patógeno

Tabela 3. Porcentagem de sementes de feijão (Phaseolus vulgaris L.) infectadas com Colletotrichum lindemuthianum, após o revestimento com polímeros e tratamento com diferentes fungicidas ${ }^{(1)}$.

\begin{tabular}{lrrrr}
\hline Tratamento $^{(2)}$ & \multicolumn{4}{c}{ Período de armazenamento (mês) } \\
\cline { 2 - 5 } & \multicolumn{1}{c}{ Antes } & \multicolumn{1}{c}{1} & \multicolumn{1}{c}{2} & \multicolumn{1}{c}{4} \\
\hline $\mathrm{T}$ & $44,00 \mathrm{Gd}$ & $38,00 \mathrm{Fb}$ & $25,50 \mathrm{Fa}$ & $43,00 \mathrm{Ec}$ \\
$\mathrm{TN}$ & $0,00 \mathrm{Aa}$ & $0,00 \mathrm{Aa}$ & $0,00 \mathrm{Aa}$ & $0,00 \mathrm{Aa}$ \\
$\mathrm{P}$ & $46,00 \mathrm{Hc}$ & $36,50 \mathrm{~Eb}$ & $23,50 \mathrm{Ea}$ & $47,50 \mathrm{Fd}$ \\
$\mathrm{B}$ & $3,00 \mathrm{Cb}$ & $0,00 \mathrm{Aa}$ & $0,00 \mathrm{Aa}$ & $0,00 \mathrm{Aa}$ \\
$\mathrm{B}+\mathrm{P}$ & $1,50 \mathrm{Bc}$ & $0,00 \mathrm{Aa}$ & $1,00 \mathrm{Bb}$ & $0,00 \mathrm{Aa}$ \\
$\mathrm{BMP}$ & $0,00 \mathrm{Aa}$ & $0,00 \mathrm{Aa}$ & $0,00 \mathrm{Aa}$ & $0,00 \mathrm{Aa}$ \\
$\mathrm{C}$ & $22,50 \mathrm{Ea}$ & $36,00 \mathrm{Dd}$ & $23,00 \mathrm{Db}$ & $30,50 \mathrm{Bc}$ \\
$\mathrm{C}+\mathrm{P}$ & $19,00 \mathrm{Da}$ & $27,50 \mathrm{Cc}$ & $23,50 \mathrm{~Eb}$ & $36,50 \mathrm{Dd}$ \\
$\mathrm{CMP}$ & $33,00 \mathrm{Fd}$ & $23,00 \mathrm{Bb}$ & $19,00 \mathrm{Ca}$ & $32,50 \mathrm{Cc}$ \\
$\mathrm{D}$ & $0,00 \mathrm{Aa}$ & $0,00 \mathrm{Aa}$ & $0,00 \mathrm{Aa}$ & $0,00 \mathrm{Aa}$ \\
$\mathrm{D}+\mathrm{P}$ & $0,00 \mathrm{Aa}$ & $0,00 \mathrm{Aa}$ & $1,00 \mathrm{Bb}$ & $0,00 \mathrm{Aa}$ \\
$\mathrm{DMP}$ & $0,00 \mathrm{Aa}$ & $0,00 \mathrm{Aa}$ & $0,00 \mathrm{Aa}$ & $0,00 \mathrm{Aa}$ \\
\hline
\end{tabular}

(1)Médias seguidas pela mesma letra, maiúscula na coluna (entre tratamentos) e minúscula na linha (entre épocas), não diferem entre si pelo teste de Scott-Knott, a 5\% de probabilidade; o CVa dos dados foi 10,43\% e o $\mathrm{CVb}, 10,77 \%$. ${ }^{(2)} \mathrm{T}$ : controle com inoculação; TN: controle sem inoculação; P: polímero; B: benomyl (pó molhável); B+P: benomyl aplicado antes dos polímeros; BMP: benomyl misturado aos polímeros; C: captan (pó seco); C+P: captan aplicado antes dos polímeros; CMP: captan misturado aos polímeros; D: carbendazin (suspensão concentrada); D+P: carbendazin aplicado antes dos polímeros; DMP: carbendazin misturado aos polímeros. 
não mais foi constatado nas sementes tratadas e revestidas. A associação dos fungicidas aos polímeros, independentemente da forma de aplicação, não comprometeu a sua eficiência de controle.

Os tratamentos com os fungicidas, associados ou não aos polímeros de revestimento, foram estatisticamente diferentes dos controles (sementes com e sem inoculação) e com níveis mais baixos de Penicillium sp., no decorrer dos quatro meses (Tabela 5). Resultados semelhantes foram obtidos em sementes de feijão tratadas com benomyl e com captan (Ellis et al., 1976). A incidência deste patógeno nas sementes tratadas ou

Tabela 4. Porcentagem de sementes de feijão (Phaseolus vulgaris L.) com bactérias e Fusarium sp., após o revestimento com polímeros e tratamento com fungicidas, antes e durante o armazenamento $^{(1)}$.

\begin{tabular}{|c|c|c|c|c|c|c|c|c|}
\hline \multirow[t]{3}{*}{ Tratamento $^{(2)}$} & \multicolumn{4}{|c|}{ Sementes com bactérias (\%) } & \multicolumn{4}{|c|}{ Sementes com Fusarium sp. (\%) } \\
\hline & \multicolumn{4}{|c|}{ Período de armazenamento (mês) } & \multicolumn{4}{|c|}{ Período de armazenamento (mês) } \\
\hline & Antes & 1 & 2 & 4 & Antes & 1 & 2 & 4 \\
\hline $\mathrm{T}$ & $4,25 \mathrm{Ca}$ & $7,00 \mathrm{~Eb}$ & $10,00 \mathrm{Dc}$ & $12,75 \mathrm{Ed}$ & $2,00 \mathrm{Cb}$ & $2,00 \mathrm{Db}$ & $0,40 \mathrm{Aa}$ & $1,60 \mathrm{Bb}$ \\
\hline $\mathrm{TN}$ & $1,75 \mathrm{Bb}$ & $0,75 \mathrm{Aa}$ & $5,75 \mathrm{Ac}$ & $5,25 \mathrm{Bc}$ & $2,10 \mathrm{Cc}$ & $0,70 \mathrm{Ba}$ & $1,40 \mathrm{Bb}$ & $0,30 \mathrm{Aa}$ \\
\hline $\mathrm{P}$ & $3,50 \mathrm{Ca}$ & $5,25 \mathrm{Cb}$ & $8,75 \mathrm{Cc}$ & $5,50 \mathrm{Bb}$ & $3,00 \mathrm{Dc}$ & $2,30 \mathrm{Db}$ & $2,30 \mathrm{Cb}$ & $1,40 \mathrm{Ba}$ \\
\hline $\mathrm{B}$ & $3,75 \mathrm{Ca}$ & $7,25 \mathrm{~Eb}$ & $10,00 \mathrm{Dc}$ & $18,00 \mathrm{Gd}$ & $2,20 \mathrm{Cb}$ & $0,00 \mathrm{Aa}$ & $0,10 \mathrm{Aa}$ & $0,00 \mathrm{Aa}$ \\
\hline $\mathrm{B}+\mathrm{P}$ & $3,25 \mathrm{Ca}$ & $4,50 \mathrm{Cb}$ & $12,50 \mathrm{Ed}$ & $7,25 \mathrm{Cc}$ & $0,40 \mathrm{Aa}$ & $0,10 \mathrm{Aa}$ & $0,20 \mathrm{Aa}$ & $0,10 \mathrm{Aa}$ \\
\hline BMP & $1,00 \mathrm{Aa}$ & $1,25 \mathrm{Aa}$ & $5,00 \mathrm{Ab}$ & $1,75 \mathrm{Aa}$ & $0,60 \mathrm{Aa}$ & $0,20 \mathrm{Aa}$ & $0,00 \mathrm{Aa}$ & $0,00 \mathrm{Aa}$ \\
\hline $\mathrm{C}$ & $6,25 \mathrm{Ec}$ & $2,25 \mathrm{Ba}$ & $7,75 \mathrm{Bd}$ & $5,00 \mathrm{Bb}$ & $2,60 \mathrm{Cc}$ & $1,80 \mathrm{Db}$ & 0,30Aa & $0,10 \mathrm{Aa}$ \\
\hline $\mathrm{C}+\mathrm{P}$ & $2,25 \mathrm{Ba}$ & $4,75 \mathrm{Cb}$ & $14,25 \mathrm{Fd}$ & $5,75 \mathrm{Bc}$ & $2,10 \mathrm{Cc}$ & $1,00 \mathrm{Bb}$ & 0,00Aa & $0,10 \mathrm{Aa}$ \\
\hline CMP & $1,75 \mathrm{Ba}$ & $4,50 \mathrm{Cb}$ & $9,25 \mathrm{Dc}$ & $8,50 \mathrm{Dc}$ & $1,50 \mathrm{Bb}$ & $1,20 \mathrm{Cb}$ & $0,20 \mathrm{Aa}$ & $0,30 \mathrm{Aa}$ \\
\hline $\mathrm{D}$ & $3,50 \mathrm{Ca}$ & $8,25 \mathrm{Fb}$ & $8,75 \mathrm{Cb}$ & $16,00 \mathrm{Fc}$ & $3,50 \mathrm{Db}$ & $0,40 \mathrm{Aa}$ & $0,00 \mathrm{Aa}$ & $0,00 \mathrm{Aa}$ \\
\hline $\mathrm{D}+\mathrm{P}$ & $0,75 \mathrm{Aa}$ & $6,25 \mathrm{Db}$ & $9,00 \mathrm{Cc}$ & $9,50 \mathrm{Dc}$ & $0,60 \mathrm{Aa}$ & $0,40 \mathrm{Aa}$ & 0,00Aa & $0,10 \mathrm{Aa}$ \\
\hline DMP & $5,25 \mathrm{Da}$ & $6,00 \mathrm{Da}$ & $15,50 \mathrm{Gc}$ & $9,00 \mathrm{Db}$ & $1,20 \mathrm{Bb}$ & $0,50 \mathrm{Aa}$ & $0,10 \mathrm{Aa}$ & $0,00 \mathrm{Aa}$ \\
\hline
\end{tabular}

${ }^{(1)}$ Médias seguidas pela mesma letra, maiúscula na coluna (entre tratamentos) e minúscula na linha (entre épocas), não diferem entre si pelo teste de Scott-Knott, a 5\% de probabilidade; o CVa dos dados de sementes com bactérias foi 24,97\% e o CVb, 22,79\%; o CVa dos dados de sementes com Fusarium sp. foi 25,64\% e o CVb, 27,45\%. ${ }^{(2)}$ T: controle com inoculação; TN: controle sem inoculação; P: polímero; B: benomyl (pó molhável); $\mathrm{B}+\mathrm{P}$ : benomyl aplicado antes dos polímeros; BMP: benomyl misturado aos polímeros; C: captan (pó seco); C+P: captan aplicado antes dos polímeros; CMP: captan misturado aos polímeros; D: carbendazin (suspensão concentrada); D+P: carbendazin aplicado antes dos polímeros; DMP: carbendazin misturado aos polímeros.

Tabela 5. Porcentagem de sementes de feijão (Phaseolus vulgaris L.) infectadas com Penicillium sp. e Eurotium sp., após o revestimento com polímeros e tratamento com diferentes fungicidas, antes e durante o $\operatorname{armazenamento}^{(1)}$.

\begin{tabular}{|c|c|c|c|c|c|c|c|c|}
\hline \multirow[t]{3}{*}{ Tratamento $^{(2)}$} & \multicolumn{4}{|c|}{ Sementes com Penicillium sp. (\%) } & \multicolumn{4}{|c|}{ Sementes com Eurotium sp. (\%) } \\
\hline & \multicolumn{4}{|c|}{ Período de armazenamento (mês) } & \multicolumn{4}{|c|}{ Período de armazenamento (mês) } \\
\hline & Antes & 1 & 2 & 4 & Antes & 1 & 2 & 4 \\
\hline $\mathrm{T}$ & $27,00 \mathrm{Ha}$ & $95,50 \mathrm{Md}$ & $35,50 \mathrm{Db}$ & $66,00 \mathrm{Fc}$ & $13,00 \mathrm{Da}$ & $17,50 \mathrm{Ib}$ & $18,00 \mathrm{Db}$ & $22,50 \mathrm{Fc}$ \\
\hline $\mathrm{TN}$ & $62,50 \mathrm{Ib}$ & $93,50 \mathrm{Ld}$ & $66,50 \mathrm{Fc}$ & $2,50 \mathrm{Ca}$ & $5,50 \mathrm{Aa}$ & $5,50 \mathrm{Ba}$ & $17,50 \mathrm{Db}$ & $17,00 \mathrm{Cb}$ \\
\hline $\mathrm{P}$ & $12,00 \mathrm{Ea}$ & $71,50 \mathrm{Jd}$ & $52,50 \mathrm{~Eb}$ & $59,50 \mathrm{Ec}$ & $12,50 \mathrm{Db}$ & $12,00 \mathrm{Fb}$ & $10,00 \mathrm{Ba}$ & $17,50 \mathrm{Cc}$ \\
\hline $\mathrm{B}$ & $13,50 \mathrm{Fc}$ & $1,00 \mathrm{Bb}$ & $0,00 \mathrm{Aa}$ & $1,00 \mathrm{Bb}$ & $12,00 \mathrm{Da}$ & $12,00 \mathrm{Fa}$ & $22,00 \mathrm{~Eb}$ & $52,00 \mathrm{Jc}$ \\
\hline $\mathrm{B}+\mathrm{P}$ & $4,50 \mathrm{Bc}$ & $3,00 \mathrm{Cb}$ & $0,50 \mathrm{Aa}$ & $0,00 \mathrm{Aa}$ & $10,00 \mathrm{Ca}$ & $19,50 \mathrm{Jb}$ & $33,50 \mathrm{Ic}$ & 41,50Id \\
\hline BMP & $12,00 \mathrm{~Eb}$ & $0,00 \mathrm{Aa}$ & $0,00 \mathrm{Aa}$ & $0,50 \mathrm{Aa}$ & $8,50 \mathrm{Ba}$ & $15,50 \mathrm{Hb}$ & $28,00 \mathrm{Gc}$ & $30,50 \mathrm{Hd}$ \\
\hline $\mathrm{C}$ & $8,50 \mathrm{Cb}$ & 21,00Id & $2,00 \mathrm{Ca}$ & $10,00 \mathrm{Dc}$ & $8,00 \mathrm{Ba}$ & $8,00 \mathrm{Da}$ & $8,00 \mathrm{Aa}$ & $13,00 \mathrm{Bb}$ \\
\hline $\mathrm{C}+\mathrm{P}$ & $15,00 \mathrm{Gc}$ & $13,00 \mathrm{~Gb}$ & $1,00 \mathrm{Ba}$ & $1,50 \mathrm{Ba}$ & $12,00 \mathrm{Db}$ & $4,00 \mathrm{Aa}$ & $29,00 \mathrm{Hd}$ & $19,50 \mathrm{Dc}$ \\
\hline CMP & $11,00 \mathrm{Dc}$ & $17,50 \mathrm{Hd}$ & $0,00 \mathrm{Aa}$ & $3,00 \mathrm{Cb}$ & $6,50 \mathrm{Aa}$ & $9,00 \mathrm{~Eb}$ & $8,50 \mathrm{Ab}$ & $12,00 \mathrm{Ac}$ \\
\hline D & $13,00 \mathrm{Fc}$ & $4,00 \mathrm{Db}$ & $0,00 \mathrm{Aa}$ & $0,00 \mathrm{Aa}$ & $6,00 \mathrm{Aa}$ & $9,50 \mathrm{~Eb}$ & $27,00 \mathrm{Fc}$ & 41,50Id \\
\hline $\mathrm{D}+\mathrm{P}$ & $4,50 \mathrm{Bc}$ & $10,00 \mathrm{Fd}$ & $0,00 \mathrm{Aa}$ & $1,00 \mathrm{Bb}$ & $15,50 \mathrm{~Eb}$ & $7,00 \mathrm{Ca}$ & $16,00 \mathrm{Cb}$ & $26,50 \mathrm{Gc}$ \\
\hline DMP & $1,50 \mathrm{Ab}$ & $6,00 \mathrm{Ec}$ & $0,00 \mathrm{Aa}$ & $0,00 \mathrm{Aa}$ & $8,50 \mathrm{Ba}$ & $13,00 \mathrm{~Gb}$ & $17,00 \mathrm{Dc}$ & $21,00 \mathrm{Ed}$ \\
\hline
\end{tabular}

(1)Médias seguidas pela mesma letra, maiúscula na coluna (entre tratamentos) e minúscula na linha (entre épocas), não diferem entre si pelo teste de Scott-Knott, a 5\% de probabilidade; o CVa dos dados de sementes com Penicillium sp. foi 30,16\% e o CVb, 32,39\%; o CVa dos dados de sementes com Eurotium sp. foi 20,38\% e o CVb, 20,61\%. ${ }^{(2)}$ T: controle com inoculação; TN: controle sem inoculação; P: polímero; B: benomyl (pó molhável); B+P: benomyl aplicado antes dos polímeros; BMP: benomyl misturado aos polímeros; C: captan (pó seco); C+P: captan aplicado antes dos polímeros; CMP: captan misturado aos polímeros; D: carbendazin (suspensão concentrada); D+P: carbendazin aplicado antes dos polímeros; DMP: carbendazin misturado aos polímeros. 
revestidas reduziu após o primeiro mês de armazenamento. O uso de polímeros como produto de revestimento, além de não ter comprometido a ação dos fungicidas sobre este fungo de armazenamento, aumentou a sua eficiência de controle, especialmente captan.

A incidência de Eurotium sp. aumentou no decorrer do armazenamento. Apesar de este fungo não ter sido controlado pelos fungicidas testados, captan mostrou melhor atuação em seu controle (Tabela 5). As sementes tratadas com benomyl, associado ou não aos polímeros, mostraram maior incidência deste patógeno, principalmente aos dois e quatro meses de armazenamento. No entanto, a associação de carbendazin com os polímeros, melhorou significativamente sua eficiência de controle, especialmente após o segundo mês.

Houve uma tendência geral de diminuição de Fusarium sp. nas sementes ao longo do armazenamento (Tabela 4), o que é considerado normal, por ser um fungo de campo (Christensen, 1957; Lázzari, 1993) e intermediário (Lázzari, 1993). Já Nascimento \& Cícero (1991) observaram comportamento contrário deste patógeno em sementes de ervilha. Até o primeiro mês, a associação dos fungicidas aos polímeros melhorou a sua eficiência no controle deste patógeno.

Alternaria sp. foi detectada em níveis de infestação muito baixos, até mesmo no controle $(1,75 \%)$, apesar de estar entre aqueles patógenos mais freqüentemente detectados em testes de sanidade de sementes de feijão
(Rolim et al., 1990). Este fungo apresentou incidência crescente ao longo dos quatro meses, conforme também observado por Nascimento \& Cícero (1991) em sementes de ervilha, e por Tuite \& Christensen (1955) em sementes de cevada armazenadas com 20\% de umidade. De acordo com Christensen (1957), a Alternaria sp. é considerada um fungo de campo e, dessa forma, não reduz a capacidade de armazenamento das sementes.

Rhizopus sp. foi detectado nas sementes de feijão em porcentagens variando de $0,00 \%$ a $72,50 \%$, com maior incidência até um mês de armazenamento (Tabela 6). Segundo Lima et al. (1984), este patógeno afeta a germinação de sementes recém-colhidas, conforme verificado em sementes de algodão não armazenadas.

Nas sementes tratadas com os fungicidas do grupo dos benzimidazóis, houve alta incidência de Rhizopus sp. (Tabela 6), semelhante aos resultados de Novo \& Menezes (1984), sugerindo ação reduzida sobre os zigomicetos. O revestimento das sementes com polímeros não prejudicou a ação de captan sobre este patógeno, cujo controle foi mais eficiente do que nos demais tratamentos.

Cladosporium sp. apresentou maior infestação nas sementes logo após a aplicação dos tratamentos (Tabela 6). Tuite \& Christensen (1955) verificaram tendência decrescente de infestação deste patógeno em sementes de cevada armazenadas com $15 \%$ a $16 \%$ de umida-

Tabela 6. Porcentagem de sementes de feijão (Phaseolus vulgaris L.) infectadas com Rhizopus sp. e Cladosporium sp., após o revestimento com polímeros e tratamento com diferentes fungicidas, antes e durante o armazenamento $^{(1)}$.

\begin{tabular}{|c|c|c|c|c|c|c|c|c|}
\hline \multirow[t]{3}{*}{ Tratamento $^{(2)}$} & \multicolumn{4}{|c|}{ Sementes com Rhizopus sp. (\%) } & \multicolumn{4}{|c|}{ Sementes com Cladosporium sp. (\%) } \\
\hline & \multicolumn{4}{|c|}{ Período de armazenamento (mês) } & \multicolumn{4}{|c|}{ Período de armazenamento (mês) } \\
\hline & Antes & 1 & 2 & 4 & Antes & 1 & 2 & 4 \\
\hline $\mathrm{T}$ & $22,00 \mathrm{Ca}$ & $34,50 \mathrm{Fb}$ & $39,00 \mathrm{Gc}$ & $33,00 \mathrm{Fb}$ & $15,00 \mathrm{Hc}$ & $4,50 \mathrm{Da}$ & $10,50 \mathrm{~Eb}$ & $18,50 \mathrm{Fd}$ \\
\hline $\mathrm{TN}$ & $44,00 \mathrm{Fa}$ & $71,50 \mathrm{Jd}$ & $59,50 \mathrm{Hc}$ & $46,00 \mathrm{~Gb}$ & $14,00 \mathrm{Gc}$ & $0,50 \mathrm{Aa}$ & $8,00 \mathrm{Db}$ & $7,50 \mathrm{Db}$ \\
\hline $\mathrm{P}$ & $22,50 \mathrm{Cb}$ & $35,50 \mathrm{Gc}$ & $40,50 \mathrm{Gd}$ & $19,00 \mathrm{Ea}$ & $24,50 \mathrm{Jc}$ & $1,50 \mathrm{Ba}$ & $26,00 \mathrm{Fd}$ & $16,50 \mathrm{~Eb}$ \\
\hline $\mathrm{B}$ & $21,00 \mathrm{Cb}$ & $22,50 \mathrm{~Eb}$ & 9,50Da & $8,00 \mathrm{Ba}$ & $10,50 \mathrm{Fc}$ & $0,50 \mathrm{Aa}$ & $2,50 \mathrm{Cb}$ & $1,00 \mathrm{Ba}$ \\
\hline $\mathrm{B}+\mathrm{P}$ & $44,00 \mathrm{Fd}$ & $37,50 \mathrm{Hc}$ & $6,00 \mathrm{Ca}$ & $12,50 \mathrm{Db}$ & $1,50 \mathrm{Cb}$ & $1,00 \mathrm{Bb}$ & $0,00 \mathrm{Aa}$ & $1,00 \mathrm{Bb}$ \\
\hline BMP & $72,50 \mathrm{Hd}$ & $18,50 \mathrm{Db}$ & $1,00 \mathrm{Ba}$ & $33,00 \mathrm{Fc}$ & $1,00 \mathrm{Bb}$ & $0,00 \mathrm{Aa}$ & $0,00 \mathrm{Aa}$ & $2,00 \mathrm{Cc}$ \\
\hline $\mathrm{C}$ & $1,50 \mathrm{Aa}$ & $15,00 \mathrm{Cc}$ & $2,00 \mathrm{Ba}$ & $3,50 \mathrm{Ab}$ & $2,00 \mathrm{Cb}$ & $0,50 \mathrm{Aa}$ & $2,00 \mathrm{Bb}$ & $2,00 \mathrm{Cb}$ \\
\hline $\mathrm{C}+\mathrm{P}$ & $19,00 \mathrm{Bc}$ & $12,00 \mathrm{Bb}$ & $0,00 \mathrm{Aa}$ & $10,50 \mathrm{Cb}$ & $0,00 \mathrm{Aa}$ & $1,00 \mathrm{Bb}$ & $0,00 \mathrm{Aa}$ & $0,00 \mathrm{Aa}$ \\
\hline CMP & $0,00 \mathrm{Aa}$ & $5,50 \mathrm{Ab}$ & $0,00 \mathrm{Aa}$ & $7,00 \mathrm{Bb}$ & $8,50 \mathrm{Ec}$ & $0,00 \mathrm{Aa}$ & $0,00 \mathrm{Aa}$ & $1,00 \mathrm{Bb}$ \\
\hline $\mathrm{D}$ & $41,50 \mathrm{Ec}$ & $42,00 \mathrm{Ic}$ & $18,50 \mathrm{Fb}$ & $7,00 \mathrm{Ba}$ & $30,50 \mathrm{Ld}$ & $4,50 \mathrm{Dc}$ & $0,50 \mathrm{Aa}$ & $2,00 \mathrm{Cb}$ \\
\hline $\mathrm{D}+\mathrm{P}$ & $56,50 \mathrm{Gc}$ & $14,50 \mathrm{Ca}$ & $20,00 \mathrm{Fb}$ & $14,00 \mathrm{Da}$ & $22,50 \mathrm{Ic}$ & $3,50 \mathrm{Cb}$ & $3,00 \mathrm{Cb}$ & $1,50 \mathrm{Ca}$ \\
\hline DMP & $36,50 \mathrm{Dc}$ & $33,00 \mathrm{Fb}$ & $14,00 \mathrm{Ea}$ & $53,00 \mathrm{Hd}$ & $4,00 \mathrm{Db}$ & $6,50 \mathrm{Ec}$ & $2,00 \mathrm{Ba}$ & $2,50 \mathrm{Ca}$ \\
\hline
\end{tabular}

${ }^{(1)}$ Médias seguidas pela mesma letra, maiúscula na coluna (entre tratamentos) e minúscula na linha (entre épocas), não diferem entre si pelo teste de Scott-Knott, a 5\% de probabilidade; o CVa dos dados de semente com Rhizopus sp. foi 51,11\% e o CVb, 55,64\%; o CVa dos dados de sementes com Cladosporium sp. foi 39,78\%, e o CVb, 33,18\%. ${ }^{(2)}$ T: controle com inoculação; TN: controle sem inoculação; P: polímero; B: benomyl (pó molhável); B+P: benomyl aplicado antes dos polímeros; BMP: benomyl misturado aos polímeros; C: captan (pó seco); C+P: captan aplicado antes dos polímeros; CMP: captan misturado aos polímeros; D: carbendazin (suspensão concentrada); D+P: carbendazin aplicado antes dos polímeros; DMP: carbendazin misturado aos polímeros. 
de, ao contrário do que aconteceu sob umidade de $20 \%$. Benomyl e captan, associados ou não aos polímeros de revestimento, apresentaram melhor controle deste patógeno, comparados ao fungicida carbendazin.

Logo após a aplicação dos tratamentos às sementes, detectou-se a presença de Peztalotia sp. no controle com inoculação $(48,5 \%)$, nas sementes revestidas com polímeros $(38,0 \%)$, e nas sementes tratadas com benomyl $(16,5 \%)$ e com benomyl misturado aos polímeros $(7,0 \%)$. Este patógeno não mais foi detectado a partir do primeiro mês de armazenamento.

\section{Conclusões}

1. O revestimento com tintas de polímeros vinílicos, associado ao tratamento químico com os fungicidas benomyl, captan e carbendazin, não interfere na germinação de sementes de feijão.

2. O revestimento com polímeros vinílicos reduz a velocidade de germinação das sementes.

3. O revestimento com polímeros vinílicos pode afetar a eficiência de controle do patógeno presente nas sementes, dependendo do fungicida utilizado e de sua forma de aplicação.

\section{Agradecimento}

Ao CNPq, pela concessão de bolsa a Jefferson Luís da Silva Costa.

\section{Referências}

AZEVEDO, L.A.S. de. Tratamento de sementes com fungicidas visando o controle de patógenos da parte aérea. In: SIMPÓSIO BRASILEIRO DE PATOLOGIA DE SEMENTES, 4., 1996, Gramado. Anais. Campinas: Fundação Cargill, 1996. p.83-91.

BACON, J.R.; CLAYTON, P.B. Protection for seeds: a new film coating technique. Span, v.29, p.54-56, 1986.

BEWLEY, J.D.; BLACK, M. Seeds: physiology of development and germination. New York: Plenum Press, 1985. 367p.

BRASIL. Ministério da Agricultura e da Reforma Agrária. Secretaria Nacional de Defesa Agropecuária. Regras para análise de sementes. Brasília, 1992. 365p.

CÁRDENAS, F.; ADAMS, M.W.; ANDERSEN, A. The genetic system for reaction of field beans (Phaseolus vulgaris L.) to infection by three physiologic races of Colletotrichum lindemuthianum. Euphytica, v.13, p.178-186, 1964.

CHRISTENSEN, C.M. Deterioration of stored grains by fungi. Botanical Review, v.23, p.108-134, 1957.
DURAN, J.M. Acondicionamento e revestimento de sementes. In: SEMINÁRIO PANAMERICANO DE SEMILLAS, 15., 1996, Gramado. Memória. Passo Fundo: Comissão Estadual de Sementes e Mudas do Rio Grande do Sul, 1998. p.107-115.

ELLIS, M.A.; GÁLVEZ, E.G.E.; SINCLAIR, J.B. Efecto de tres fungicidas sobre la germinación de semilla infectada de fríjol (Phaseolus vulgaris). Turrialba, v.26, p.399-402, 1976.

LANZA, M.A.; PAULA JÚNIOR, T.J.; VINHADELLI, W.S.; MORANDI, M.A.B.; BARROS, E.G.; MOREIRA, M.A. Resistência à antracnose em cultivares de feijoeiro-comum recomendadas para Minas Gerais. Fitopatologia Brasileira, v.22, p.560-562, 1997.

LÁZZARI, F.A. Umidade, fungos e micotoxinas na qualidade de sementes, grãos e rações. Curitiba: Edição do Autor, 1993. $140 \mathrm{p}$.

LIMA, E.F.; VIEIRA, R.M.; CARVALHO, J.M.F.C. Influência de Rhizopus sp., Aspergillus niger e A. flavus na deterioração de sementes de algodoeiro armazenadas. Fitopatologia Brasileira, v.9, p.555$560,1984$.

MAUDE, R. Progressos recentes no tratamento de sementes. In: SEMINÁRIO PANAMERICANO DE SEMILLAS, 15., 1996, Gramado. Memória. Passo Fundo: Comissão Estadual de Sementes e Mudas do Rio Grande do Sul, 1998. p.99-106.

MENEZES, J.R. de; MOHAN, S.K. Efeito da seleção visual da semente de feijão (Phaseolus vulgaris L.) sobre a qualidade sanitária. In: REUNIÃO NACIONAL DE PESQUISA DE FEIJÃO, 1., 1982, Goiânia. Anais. Goiânia: Embrapa-CNPAF, 1982. p.343-344. (Documentos, 1).

NASCIMENTO, W.M.; CÍCERO, S.M. Qualidade de sementes de ervilha tratada com fungicida - I: qualidade sanitária. Revista Brasileira de Sementes, v.13, p.5-12, 1991.

NOVO, R.J.; MENEZES, M. Eficiência de fungicidas no tratamento de sementes de sorgo granífero, Sorghum bicolor. Fitopatologia Brasileira, v.9, p.543-549, 1984.

ROLIM, P.R.R.; CENTURION, M.A.P.C.; MENTEN, J.O.M. Alternaria sp. em feijoeiro (Phaseolus vulgaris): incidência na semente, tipos morfológicos, patogenicidade e transmissibilidade de diferentes isolados. Summa Phytopatologica, v.16, p.130-139, 1990.

SARTORATO, A. Fungicidas recomendados para tratamento de sementes de feijão. In: SIMPÓSIO BRASILEIRO DE PATOLOGIA DE SEMENTES, 4., 1996, Gramado. Anais. Campinas: Fundação Cargill, 1996. p.45-51.

SAUVE, E.M.; SHIEL, R.S. Coating seeds with resins. Journal of Horticultural Science, v.55, p.371-373, 1980.

SCOTT, A.J.; KNOTT, M.A. Cluster analysis method for grouping means in the analysis of variance. Biometrics, v.30, p.507-512, 1974.

TUITE, J.F.; CHRISTENSEN, C.M. Grain storage studies XVI: influence of storage conditions upon the fungus flora of barley seed. Cereal Chemistry, v.32, p.1-11, 1955.

Recebido em 16 de julho de 2003 e aprovado em 11 de maio de 2004 